ously. But where the gouge has to be used the joint movements are less free, and there is a tendency to ankylosis.

Notwithstanding, this operation is a great gain when the dislocation is single or, after good motion has been established on one side, when dislocation is double. When consolidation has taken place, and a new articulation has been formed, as in Fournier's case I never interfere; but at any period before consolidation is complete, the head of the femur may be drawn down after the trochanteric muscles have been divided, and with or without the gouge, the head of the femur may be safely and permanently restored to the acetabulum.

\section{AN AN'TISEPTIC TABLET FOR NASAL WASH.} BY JOHN EDWIN RHODES, M.D. CHICAGO.

The necessity of a cleansing and an antiseptic wash for the nasal cavities is indicated in a variety of conditions. In cases of simple chronic catarrhal inflammation in which there is an excess of mucus which gathers and forms crusts in the cavities, of ten due to a slight nasal obstruction and accompanied with an unpleasant "catarrhal odor," the indications for treatment are often chiefly fulfilled by some antiseptic wash which will effectually dislodge the secretions and counteract the odor. In another class of cases instead of obstruction, we find an enlarged passage, atrophy of the membrane, and sometimes of the turbinated bones. Here even an exaggerated expiratory blast of air is inefficient in dislodging the secretion. This rapidly dries upon the surface of fine membrane, decomposes and causes pathologic changes in the tigsues, and the patient gives as one of the most distressing features of the condition the bad odor that accompanies it. Here there is an indication for an agent that will both cleanse the nasal passages, dis. infect them and stimulate the mucous glands. After operations of various kinds, as the removal of exostoses, the cauterization of the membrane, the removal of polypi, tumors, etc., an agent is needed that will render the parts thoroughly aseptic until healing has taken place. In operations about the fauces, the naso-pharynx, the pharynx, the tonsils, it is always advisable to use some strongly antiseptic solution as a spray or gargle for a few days succeed. ing the operation.

To meet such indications as have been briefly enu. merated above the following formula has been found to possess the requisite agents in suitable proportions. The tablets can be employed in all conditions, and are unirritating if used in the strength of one tablet in from one-half to two-thirds of a glass of lukewarm water. In cleansing the nares it is much to be preferred that the patient should draw the water gently through the passages from the hand than to use auy kind of a douche, as the latter in very many cases is improperly used, and the liquid is carried through the nasal passages with such force as to do local damage and perhaps reach the middle ear through the Eustachian tubes and set up a middle ear.inflammation. This formula has been thoroughly tested by use in a large number of cases in private practice in the last two years, and is more satisfac. tory than any agents $I$ have ever used for like purposes.

It has been put up in the form of compressed tab- lets, which, as prepared by Gale \& Blocki, are readily soluble, and in every way satisfactory. Each tablet contains the amount indicated in the formula.

DR. RHODES' NASAL TALLETS.

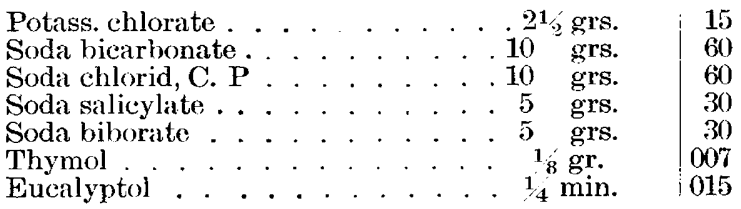

MYRINGITIS ACUTA-MYRINGITIS BULLOSA. BY FRANK ALLPORT, M.D.

PHOFESSOK OF CIINICAI OPHTHALMOLOGY AND OTOLOGY IN THE MINNESOTA STATE UNIVERSITY, PRESIDENT OF THE MINNESOTA STATE MEDICAL SOCIETY, ETC. MINNEAPOLIS, MINN

Miss M. C., age 20; left ear; general health not good; has had naso-pharyngeal colds for two or three weeks, accompanied by a hard cough. Nov. 11, 1895, at about 11 P.M., experienced suddenly a severe earache, which continued with increasing severity till the morning of November 12, when I was consulted. I found the view of the membrana tympanum obstructed by three large bullæ situated as follows: One at the upper anterior quadrant, extending down nearly to the inferior extremity of the handle of the malleus. This was large and deeply congested, the redness passing on to the meatal wall, as it joins the drum-head.

Another was situated at the junction of the drumhead with the posterior meatal wall, and was more on the latter than the former. This was also much inflamed and large. Another blister occurred exactly at the inferior extremity of the malleus, and was smaller than the others-quite white and not inflamed. A cluster of prominent blood-vessels extended from this to the posterior blister. The bullæ were separate and distinct, for $I$ punctured all three, as each one required a separate incision to liberate its individual serous fluid. I was careful not to penetrate beneath the dermic layer of the membrane, and a thorough inflation of the middle ear with the catheter and air bag after the puncture was made evidenced an intact condition of the underlying membranic coats. The hearing in this ear was somewhat but not markedly reduced. The case was seen by Dr. W. N. Porteus, of this city, at my request.

November 13 , I found the blisters all thoroughly collapsed; some membranic inflammation, no pain, slept well, hearing almost completely restored. November 19, drum-head fully restored to a normal appearance and hearing perfect.

THE DEGENERATE EAR.

Read in the Section on Neurology and Medical Jurisprudence, at the Forty-sixth Annual Meeting of the American Medical Association, at Baltimore, Md., May 7-10, 1895 .

BY EUGENE S. TALBOT, M.D.

FELLOW OF THE CHICAGO ACADEMY OF MEDICINE. CHICAGO.

(Concluded from page 58.)

Figures 10, 11 and 12 illustrate the different stages of excessive and arrested development of the helix. For the purpose of giving a better description of the stigmata of the ear I have divided the ear into upper, middle and lower third. Figure 10 shows arrest of the helix as far up as the upper third, while the upper part is well developed. Figure 11 shows the helix normally developed. Figure 12 illustrates the lower 